\title{
Cytoplasmic PTEN protein loss distinguishes intraductal carcinoma of the prostate from high-grade prostatic intraepithelial neoplasia
}

Tamara L Lotan ${ }^{1,2}$, Berrak Gumuskaya ${ }^{1}$, Hameed Rahimi ${ }^{1}$, Jessica L Hicks ${ }^{1}$, Tsuyoshi Iwata ${ }^{3}$, Brian D Robinson ${ }^{1}$, Jonathan I Epstein ${ }^{1,2,4}$ and Angelo M De Marzo ${ }^{1,2,4}$

${ }^{1}$ Department of Pathology, Johns Hopkins Medical Institutions, Baltimore, MD, USA; ${ }^{2}$ Department of Oncology, Johns Hopkins Medical Institutions, Baltimore, MD, USA; ${ }^{3}$ Department of Urology, Kyoto Prefectural University, Kyoto, Japan and ${ }^{4}$ Department of Urology, Johns Hopkins Medical Institutions, Baltimore, MD, USA

Intraductal carcinoma of the prostate is a marker of aggressive disease. However, intraductal carcinoma exists on a morphologic continuum with high-grade prostatic intraepithelial neoplasia (PIN) and distinguishing intraductal carcinoma from PIN is a common diagnostic dilemma with significant clinical implications. We evaluated whether immunostains for PTEN and ERG can sensitively identify intraductal carcinoma and accurately distinguish it from high-grade PIN. A combined immunostain for PTEN, ERG, p63 and CK903 was developed and validated. Radical prostatectomy specimens with lesions meeting criteria for intraductal carcinoma $(n=45)$, intraductal cribriform proliferations falling short of intraductal carcinoma $(n=15)$, and PIN lesions $(n=39)$ were retrospectively identified and assessed for PTEN and ERG. Cytoplasmic PTEN loss was identified in $84 \%(38 / 45)$ of the intraductal carcinoma and $100 \%(15 / 15)$ of intraductal cribriform proliferation cases. In contrast, cytoplasmic PTEN loss was never observed in PIN (0/39; $P<0.0001)$. Of the 53 cases of intraductal carcinoma or intraductal cribriform proliferation with cytoplasmic PTEN loss, it was homogeneously lost in 42 cases (79\%). Weak, focal nuclear positivity for PTEN was retained in 31 of these 42 cases (74\%). ERG expression was identified in 58\% (26/45) of intraductal carcinoma and $67 \%(10 / 15)$ of intraductal cribriform proliferations compared with 13\% (5/39) of PIN. Concordance between the PTEN/ERG status of the intraductal carcinoma lesions and the concurrent invasive carcinoma was high ( $>95 \%$ and $P<0.0001$ for each), and substantially less for PIN and the concurrent invasive tumor (83\% for PTEN and $67 \%$ for ERG; $P=N S$ for each). Cytoplasmic PTEN loss occurs in the majority of intraductal carcinoma and intraductal cribriform proliferation cases. Cytoplasmic PTEN loss was never observed in PIN (100\% specificity). Our study identifies PTEN loss as a potentially useful marker to distinguish intraductal carcinoma from PIN and provides a plausible molecular explanation for why intraductal carcinoma is associated with poor prognosis.

Modern Pathology (2013) 26, 587-603; doi:10.1038/modpathol.2012.201; published online 7 December 2012

Keywords: ERG; immunohistochemistry; intraductal carcinoma; prostatic adenocarcinoma; PTEN

Intraductal carcinoma of the prostate (IDC-P) is an excellent marker of clinically aggressive disease. Since its original description, numerous studies have confirmed that intraductal carcinoma is almost invariably associated with high-grade invasive carcinoma in radical prostatectomy specimens. ${ }^{1-7}$ Even when identified on biopsy as an isolated lesion

Correspondence: Dr TL Lotan, MD, Department of Pathology, Johns Hopkins Medical Institutions, 855N Wolfe Street, Rangos 454, Baltimore, MD 21205, USA.

E-mail: tlotan1@jhmi.edu

Received 11 July 2012; revised 11 October 2012; accepted 12 October 2012; published online 7 December 2012 without invasive tumor, because of its frequent association with unsampled concurrent high-grade tumor, ${ }^{8,9}$ it has been recommended that men with intraductal carcinoma should undergo definitive treatment. Finally, two recent studies demonstrate that identification of intraductal carcinoma in needle biopsies containing invasive tumor also predicts for worse clinicopathologic outcomes, even after accounting for the Gleason grade and extent of the invasive component. ${ }^{10,11}$

Importantly, intraductal carcinoma exists along a morphologic spectrum. At one end, intraductal carcinoma shows significant morphologic overlap with cribriform high-grade invasive 
adenocarcinoma, and since the advent of basal cell immunostains, it has been increasingly appreciated that as many as $30 \%$ of cribriform carcinomas have an intraductal component. ${ }^{12}$ At the other end of the spectrum, however, intraductal carcinoma lesions can be more difficult to recognize and many have significant morphologic overlap with isolated highgrade prostatic intraepithelial neoplasia (PIN), a lesion that is not by itself an indication for treatment or even clinical follow-up in most cases. ${ }^{3,4,7-9}$ As the diagnoses of intraductal carcinoma and high-grade PIN have such different implications for patient care and prognosis, intraductal carcinoma is currently identified on needle biopsy using a strict set of morphologic criteria designed to avoid its overdiagnosis. ${ }^{8}$ Since these criteria were intentionally created to be specific for intraductal carcinoma, they likely lack sensitivity and may result in the underdiagnosis of clinically significant intraductal tumors. Recently, along with others, we have identified a substantial group of intraductal cribriform proliferative lesions that do not formally qualify as intraductal carcinoma using current morphologic criteria, but are more concerning in terms of extent of involvement and/or cytologic atypia than typical high-grade PIN. ${ }^{7}$ We have called these intraductal cribriform proliferations, where the differential diagnosis is between high-grade PIN and intraductal carcinoma (TLL and JIE, unpublished data). Management of these lesions remains uncertain.

Given the importance of distinguishing intraductal carcinoma from high-grade PIN and the uncertainties associated with the current morphologic classification system, an ancillary test based on molecular alterations present in intraductal carcinoma and not high-grade PIN would help identify these intraductal proliferative lesions with more accuracy. Importantly, current data suggest that intraductal carcinoma may be separable from highgrade PIN at the molecular level. Although pathologists have long debated whether intraductal carcinoma represents a de novo intraductal lesion or late colonization of benign ducts by high-grade invasive tumor, ${ }^{1-9}$ the frequent association of intraductal carcinoma with concurrent and often physically adjacent invasive tumor suggests the latter etiology is most common. ${ }^{9}$ Indeed, in the only molecular studies of intraductal carcinoma to date, it had a markedly higher rate of loss of heterozygosity (LOH) and $E R G$ gene rearrangement than high-grade PIN, ${ }^{6,13-15}$ and a rate even higher than that seen in invasive carcinoma in some cases. Given that isolated high-grade PIN is the presumptive precursor lesion to many invasive carcinomas, these data suggest that intraductal carcinoma is molecularly more similar to invasive high-grade carcinoma and may be distinguished from highgrade PIN using appropriate molecular-based tools.

In order to develop an immunohistochemical (IHC) test to distinguish intraductal carcinoma and high-grade PIN, we took advantage of the fact that $E R G$ gene rearrangements and deletions involving the PTEN locus are common molecular changes identified in invasive prostatic carcinoma and these changes occur much more infrequently in high-grade PIN. ${ }^{16-35} E R G$ gene rearrangements occur in $40-60 \%$ of surgically treated invasive prostatic adenocarcinoma and have been identified in $<20 \%$ of cases of high-grade PIN. ${ }^{23,24,36-38}$ PTEN loss occurs in $30-70 \%$ of tumors, and is also quite rare in high-grade PIN. ${ }^{25-35}$ As both genetic changes can be sensitively detected with previously validated IHC assays, ${ }^{35,39-42}$ we sought to determine whether we could develop an easily applied IHC test for PTEN and ERG that might help to distinguish intraductal carcinoma from high-grade PIN.

\section{Materials and methods}

\section{Patient and Tissue Selection}

This study, including tissue collection and IHC staining, was approved by the Johns Hopkins Hospital Institutional Review Board. Radical prostatectomy specimens with lesions meeting criteria for intraductal carcinoma $(n=45)$, intraductal cribriform proliferations where the differential diagnosis was intraductal carcinoma vs high-grade PIN (hereafter referred to as 'intraductal cribriform proliferation', $n=15)$, or high-grade PIN $(n=39)$ were classified on a hematoxylin and eosin-stained sections by two uropathologists (JIE and TLL) blinded to the immunostaining results and using previously published morphologic criteria. ${ }^{8}$ Table 1 identifies the morphologic characteristics of the selected intraductal carcinoma and intraductal cribriform proliferation cases. High-grade PIN was identified using standard criteria as an intraductal proliferation with tufting, or at times micropapillary, architecture, absence of cribriform architecture and with nucleoli easily visible at $\times 20$ magnification. Cases showing flat high-grade PIN were not included. Exclusion criteria for PIN also included presence of concurrent intraductal carcinoma in the

Table 1 Morphologic criteria for classification of intraductal lesions

\begin{tabular}{lc} 
Morphologic criteria for classification \\
\hline Intraductal carcinoma (n=45) \\
$\quad$ Micropapillary with marked atypia & $3(7 \%)$ \\
Micropapillary with necrosis & $2(4 \%)$ \\
Loose cribriform with marked atypia & $0(0 \%)$ \\
Loose cribriform with necrosis & $4(9 \%)$ \\
Dense cribriform & $30(67 \%)$ \\
Solid & $6(9 \%)$ \\
& \\
Intraductal cribriform proliferation (n=15) & $15(100 \%)$ \\
Loose cribriform without marked atypia or & \\
necrosis &
\end{tabular}


same case. All intraductal cribriform proliferation and high-grade PIN lesions were identified in radical prostatectomy specimens occurring at the Johns Hopkins Hospitals (Baltimore, MD, USA). Of the intraductal carcinoma cases, 67\% (30/45) were selected from radical prostatectomy specimens in the surgical pathology files of the Johns Hopkins Hospitals, while an additional 10 cases were retrospectively selected from a previously reported highrisk group of patients enrolled in an adjuvant trial of docetaxel who underwent surgery at an outside institution. ${ }^{43}$ Finally, five intraductal carcinoma cases were identified by prospectively following a previously reported group of patients with isolated intraductal carcinoma on needle biopsy and subsequent radical prostatectomy at an outside institution. ${ }^{9}$ In all, 96\% (43/45) of intraductal carcinoma cases and $100 \%$ (15/15) of the intraductal cribriform proliferation cases had an associated invasive tumor component present on the same section as the intraductal lesion, whereas 2 cases of intraductal carcinoma occurred as isolated lesions without any invasive tumor in the radical prostatectomy. Clinicopathologic characteristics of the three patient groups are recorded in Table 2.

\section{Immunohistochemistry}

Validation of the individual IHC protocols for PTEN and ERG has been previously published. ${ }^{35,42}$ Here, to simultaneously interrogate the status of ERG, PTEN and basal cells in tissue sections, we developed a novel 3 color chromogenic immunostain for PTEN, ERG, p63 and 34 $\beta$ E12 (CK903). In this assay, basal cells (p63 and 34 $\beta$ E12) are labeled in red (alkaline phosphatase (AP) using Vector Red as chromagen), PTEN is labeled in brown (horseradish peroxidase (HRP) using 3,3' diamino benzidine (DAB) as chromagen) and ERG is labeled in purple (HRP using Vector VIP purple as chromogen). To validate this multi-labeling assay

Table 2 Pathologic stage and grade of invasive tumor associated with intraductal lesion

Pathologic stage and grade of invasive tumor

\begin{tabular}{lccc}
\hline & $\begin{array}{c}\text { Intraductal } \\
\text { carcinoma } \\
(\mathrm{n}=45)\end{array}$ & $\begin{array}{c}\text { Intraductal } \\
\text { cribriform } \\
\text { proliferation } \\
(\mathrm{n}=15)\end{array}$ & $\begin{array}{c}\text { High- } \\
\text { grade } \\
\text { PIN } \\
(\mathrm{n}=39)\end{array}$ \\
\hline IDC-P only & $2(4 \%)$ & $0(0 \%)$ & $0(0 \%)$ \\
pT2 & $5(11 \%)$ & $4(27 \%)$ & $15(38 \%)$ \\
pT3A & $15(33 \%)$ & $4(27 \%)$ & $16(41 \%)$ \\
pT3B & $14(31 \%)$ & $4(27 \%)$ & $6(15 \%)$ \\
pTXN1 & $9(20 \%)$ & $3(20 \%)$ & $2(5 \%)$ \\
Gleason 6 & $2(4 \%)$ & $1(7 \%)$ & $3(8 \%)$ \\
Gleason 7 & $20(44 \%)$ & $12(80 \%)$ & $25(64 \%)$ \\
Gleason $8-10$ & $21(47 \%)$ & $2(13 \%)$ & $11(28 \%)$ \\
\hline
\end{tabular}

in terms of sensitivity and specificity, we performed a number of control experiments on adjacent slides of known PTEN, ERG and basal cell status and found that with our optimized assay the results from the multi-labeling were virtually identical to that of the individual IHC assays. We further demonstrated that there were no cross-reactions between secondary antibodies or enzymes by performing control experiments in which individual primary or secondary antibodies were omitted. Following optimization, we arrived at the following set of conditions (with 5-min washes in TBST between each step). Antigen unmasking was performed by steaming in EDTA buffer (pH 8.0) for $45 \mathrm{~min}$. Nonspecific binding was blocked by incubating in dual blocker HP/AP solution (DAKO; Carpinteria, CA, USA) for $5 \mathrm{~min}$ at room temperature, followed by Quanto UV Block (Ultravision Quanto; ThermoScientific, Waltham, MA, USA) for $5 \mathrm{~min}$ at room temperature. Slides were incubated with an anti-PTEN (rabbit monoclonal; clone D4.3, \#9188, 1:50; Cell Signaling Technologies, Beverly, MA, USA)/anti-ERG (mouse monoclonal; CM421C; 1:50; BioCare Medical, Concord, CA, USA) antibody cocktail for $45 \mathrm{~min}$ at room temperature. A HRPlabeled anti-rabbit polymer (PowerVision Poly-HRP Anti-Rabbit IgG; Leica Microsystems, Bannockburn, IL, USA) was then applied for $30 \mathrm{~min}$ at room temperature. Signal detection for PTEN (brown) was then performed for $20 \mathrm{~min}$ at room temperature using 3,3'-diaminobenzidine tetrahydrochloride (DAB) as the chromagen. This was followed by application of a HRP-labeled anti-mouse polymer (Ultravision Quanto anti-mouse IgG; ThermoScientific) and signal detection using the Vector VIP kit according to the manufacturer's instructions (Vector Labs, Burlingame, CA, USA), which results in purple staining for ERG. Then, slides were incubated with an anti-TP63 (mouse; \#NB100-691; 1:50; Novus BIologicals, Littleton CO, USA)/ cytokeratin 903 (mouse; \#ENZ-C34903; 1:50, Enzo Life Sciences, Farmingdale, NY, USA) cocktail for $45 \mathrm{~min}$ at room temperature. Finally, signal detection for p63 and CK903 was performed using an AP-labeled anti-mouse polymer (PowerVision Poly-AP Mouse IgG; Leica Microsystems), which results in red immunolableing. Slides were then dehydrated, mounted and coverslipped. Counterstaining was not performed to optimize visualization of the Vector VIP chromogen.

\section{Interpretation of IHC}

Cytoplasmic PTEN and nuclear ERG protein were visually scored using a previously validated dichotomous scoring system ${ }^{35,42}$ by two urologic pathologists (TLL and AMD). All lesional glands on the standard slide were scored (minimum 5, up to 50) once they met morphologic criteria for intraductal carcinoma, intraductal cribriform proli- 
feration or high-grade $\mathrm{PIN},{ }^{8}$ based on side-by-side comparisons with a hematoxylin- and eosin-stained section. In addition, lesions were scored only if the presence of basal cells could be documented on the quadruple-immunostained section. Using the previously validated system, lesional tissue was scored as negative or positive for PTEN protein by comparing cytoplasmic staining in malignant glands with that of adjacent benign glands and/or stroma, which provided an internal positive control within each tissue section. Cytoplasmic staining for PTEN was classified as homogeneously negative if the intensity was markedly decreased or entirely negative across $>90 \%$ of lesional epithelial cells within each gland when compared with the surrounding benign glands and/or stroma. Cytoplasmic staining for PTEN was considered homogeneously positive if cytoplasmic staining was present in $>90 \%$ of lesional cells. This dichotomous scoring system was derived and validated in our previous study of PTEN IHC using the same antibody as the current study and a similar staining protocol. ${ }^{35}$ In that study, we found that using this scoring system, PTEN IHC was 100\% sensitive and $97.8 \%$ specific for PTEN genomic loss across a panel of 58 cell lines. The assay was also found to be between 75 and $86 \%$ sensitive for PTEN genomic loss in 119 genetically characterized prostate tumor tissues. Importantly, as has been previously reported for PTEN fluorescence in situ hybridization (FISH), a number of cases in the current study showed heterogeneous PTEN protein expression. Cytoplasmic staining for PTEN was classified as heterogeneous if $>10$ and $<90 \%$ of lesional cells within a single gland showed positive cytoplasmic staining compared with the internal control (Figure 1c) or if some lesional glands were classified as negative for PTEN protein ( $>90 \%$ of cells markedly decreased) and some positive for PTEN protein ( $<10 \%$ of cells markedly decreased). Staining for nuclear ERG was assessed in comparison with stromal endothelial cell staining, which provided an internal positive control for ERG in each section. Similarly, adjacent benign glands provided an internal negative control for ERG staining in all cases. Using cutoffs found to be nearly $90 \%$ specific for $E R G$ gene rearrangement in a prior study by our group, ${ }^{42}$ staining for ERG was considered positive if any lesional cells showed nuclear positivity even those with somewhat weaker staining when compared with surrounding endothelial cells, and negative if no lesional cells were positive.

\section{Statistical Analysis}

Fisher's exact tests were used to determine the correlation of PTEN and ERG protein expression with one another and with morphologic characteristics of the intraductal proliferation.

\section{Results}

\section{Morphologic Characteristics of Cases Studied}

Radical prostatectomy specimens $(n=45)$ containing foci of intraductal carcinoma were identified by two uropathologists (JIE and TLL) using H\&Estained sections and blinded to immunostaining results. Cases of intraductal carcinoma were classified by applying previously established morphologic criteria. ${ }^{8}$ The most common criterion used for classifying a case as intraductal carcinoma was dense cribriform architecture of involved glands ( $n=30,67 \%$ of cases), which was defined as a cribriform gland with $>50 \%$ filling by epithelial cells (Table 1; Figures 1a, b and d). Of these dense cribriform cases, $53 \%(n=16)$ had a component of loose cribriform architecture as well, which was defined as a cribriform gland with $<50 \%$ filling by epithelial cells (Figures 1c and 2b). Cases with predominant micropapillary architecture were relatively rare ( $n=5$, or $11 \%$ of cases; Figures 2a and c) as was predominant solid architecture $(n=6$ or $14 \%$ of cases). In all, 96\% (43/45) of intraductal carcinoma cases were associated with invasive tumor, whereas the remaining 2 cases (4\%) occurred as isolated lesions in the radical prostatectomy specimen. Both of these radical prostatectomies were indicated by a biopsy showing intraductal carcinoma without associated invasive tumor. In all cases, the invasive tumor component was intimately associated with the intraductal carcinoma lesions on the same tissue section and was within $3 \mathrm{~mm}$ distance of the intraductal carcinoma when present.

Intraductal cribriform proliferation cases $(n=15)$ were similarly identified in a blinded manner as cases in which there was an intraepithelial proliferation with loose cribriform architecture, but lacking marked cytologic atypia (nuclei $<6 \times$ size of stromal nuclei) and comedonecrosis. All cases (15/15, 100\%) were intimately associated with invasive tumor (within $3 \mathrm{~mm}$ distance) on the same tissue section. High-grade PIN cases were identified using standard histologic criteria with nucleoli easily visible at $\times 20$ magnification). Cases with concurrent intraductal carcinoma were excluded from this group. All of the high-grade PIN cases (39/39, 100\%) contained concurrent invasive tumor in the prostatectomy specimen with 59\% (23/39) containing high-grade PIN within $3 \mathrm{~mm}$ of the invasive tumor foci and 41\% (16/39) containing high-grade PIN $>3 \mathrm{~mm}$ away from the invasive tumor foci.

\section{Clinicopathologic Characteristics of Cases Studied}

Consistent with previous studies, ${ }^{1-5,8,44}$ radical prostatectomy cases with intraductal carcinoma also contained invasive tumor with generally highrisk pathologic features (Table 2). Of intraductal carcinoma cases, 84\% (38/45) contained invasive 
tumor at pathologic stage pT3A or higher and 91\% (41/45) were Gleason score 7 or higher. Cases containing intraductal cribriform proliferation showed similarly high-risk pathologic features at radical prostatectomy, with $73 \%(11 / 15)$ containing invasive tumor at stage pT3A or higher and $93 \%$

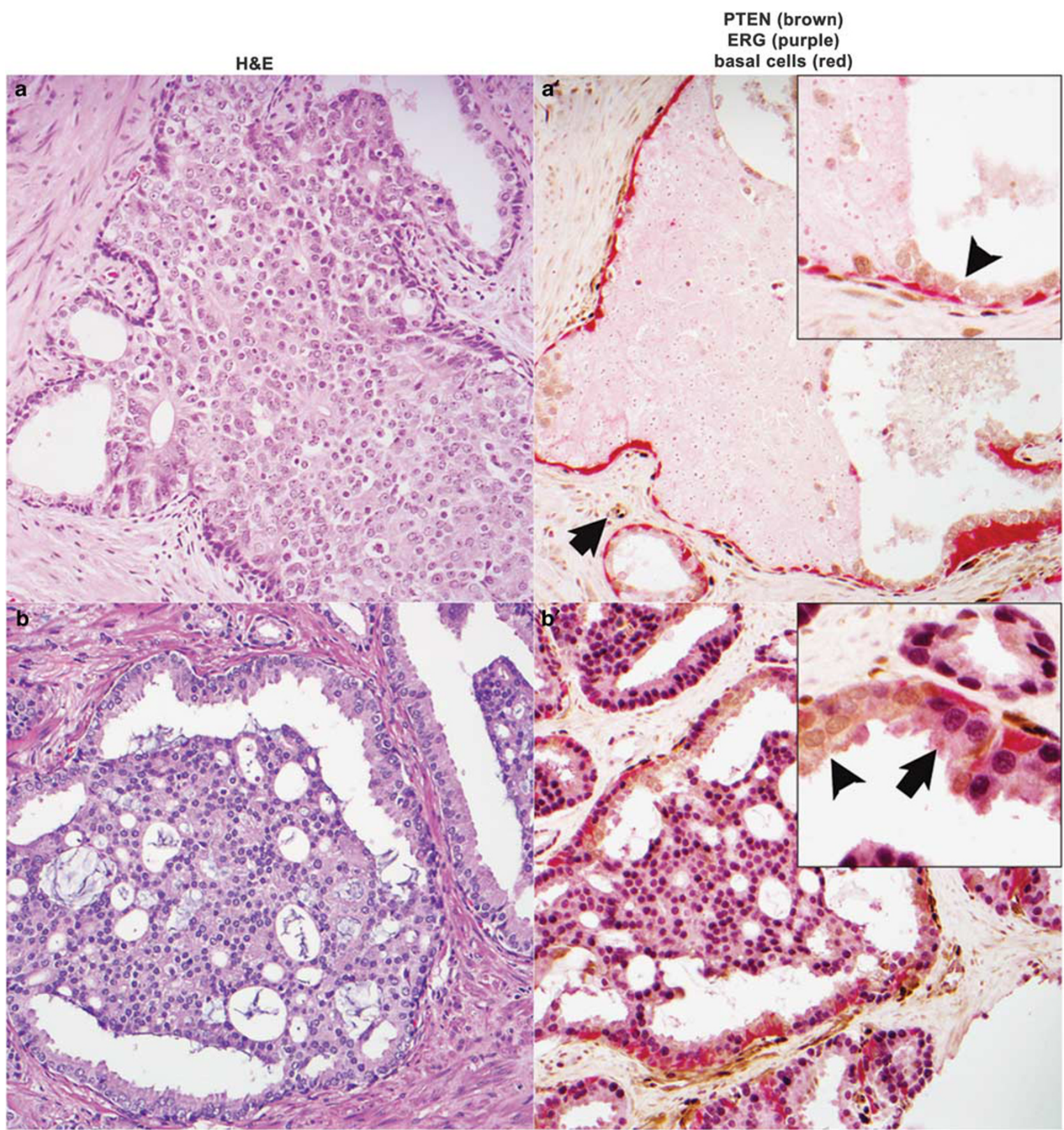

Figure 1 Cytoplasmic PTEN protein loss and nuclear ERG protein expression in intraductal carcinoma lesions. (a, a') Homogeneous cytoplasmic PTEN protein loss without ERG expression in a solid to dense cribriform intraductal carcinoma lesion. PTEN protein is entirely absent in the lesional cells, but retained in the cytoplasm and nucleus (brown) of the cytologically benign luminal cells lining the prostatic duct (inset, arrowhead). Basal cells are highlighted by p63-CK903 immunostaining (red). Nuclear ERG is present in adjacent benign endothelial cells (arrow). (b, b') Cytoplasmic PTEN protein loss with nuclear ERG expression in a dense cribriform intraductal carcinoma lesion. Cytoplasmic PTEN (brown) is expressed in retained benign luminal epithelial cells within the involved duct (inset, arrowhead), but is absent in adjacent, ERG-positive cells (inset, arrow). Basal cells are highlighted by p63-CK903 immunostaining (red). (c, c') Heterogeneous cytoplasmic PTEN protein loss in intraductal carcinoma with loose cribriform morphology. Cytoplasmic PTEN protein is retained in a subset of cytologically atypical cells. Cells with PTEN loss have more abundant cytoplasm (black arrow) and are less crowded than areas of the gland with PTEN retention (arrowhead). Surrounding invasive tumor (note absent basal cell layer in red) demonstrates cytoplasmic PTEN protein loss (gray arrow). (d, d') Homogeneous PTEN protein expression in dense cribriform to solid focus of intraductal carcinoma. 


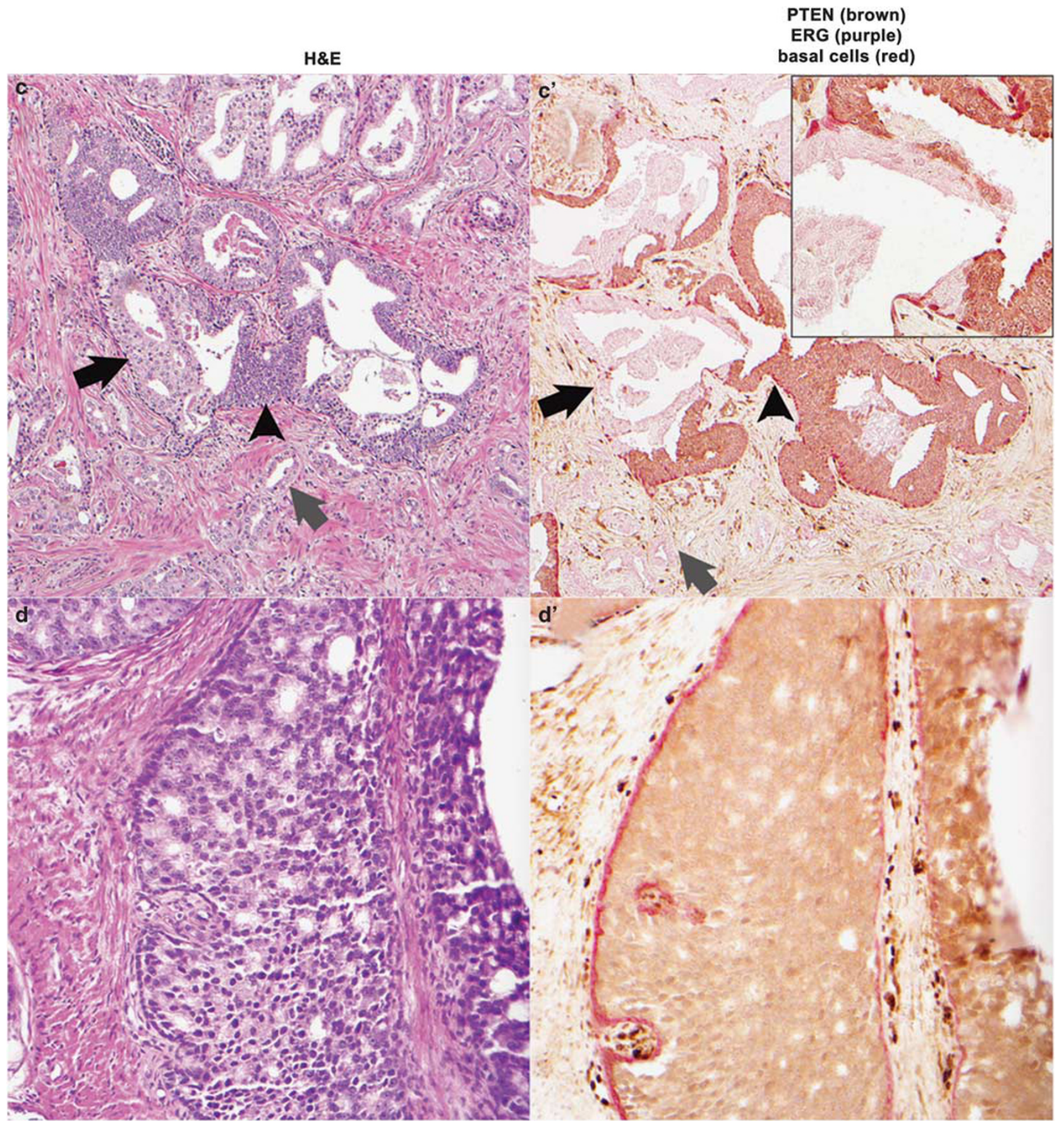

Figure 1 Continued.

(14/15) at Gleason score 7 or higher. High-grade PIN cases were selected to be roughly matched with intraductal carcinoma cases in terms of grade and stage, and thus $61 \%(24 / 39)$ were pT3A or higher and $92 \%(36 / 39)$ were Gleason score 7 or higher. As cases containing concurrent intraductal carcinoma were excluded from inclusion in the high-grade PIN control group (thus excluding a substantial number of high-stage/grade cases) it was not possible to completely match the high-grade PIN and intraductal carcinoma groups in terms of stage and grade (Table 2).

\section{Cytoplasmic PTEN Protein Expression in Intraductal Lesions}

PTEN protein was uniformly expressed in benign prostate tissue and this served as an internal positive control in every case. Consistent with our previous studies, PTEN protein was diffusely expressed in the cytoplasm and nucleus of benign luminal and basal epithelial cells, as well as in the surrounding stromal tissue. Overall, 84\% (38/45) of intraductal carcinoma cases showed cytoplasmic PTEN loss in at least a component of the intraductal 
tumor, with 64\% (29/38) showing uniform loss in all involved glands (Figures $1 \mathrm{a}$ and $\mathrm{b}$ ), and 20\% (9/38) showing heterogeneous cytoplasmic PTEN expression where some lesional cells showed cytoplasmic PTEN loss (Figure 1c) and some cells showed normal PTEN expression (Table 3a). Of note, 50\% $(1 / 2)$ of cases with isolated intraductal carcinoma on radical prostatectomy (no concurrent invasive tumor) showed homogeneous PTEN protein loss. Overall, only $16 \%$ of cases (7/45) retained PTEN

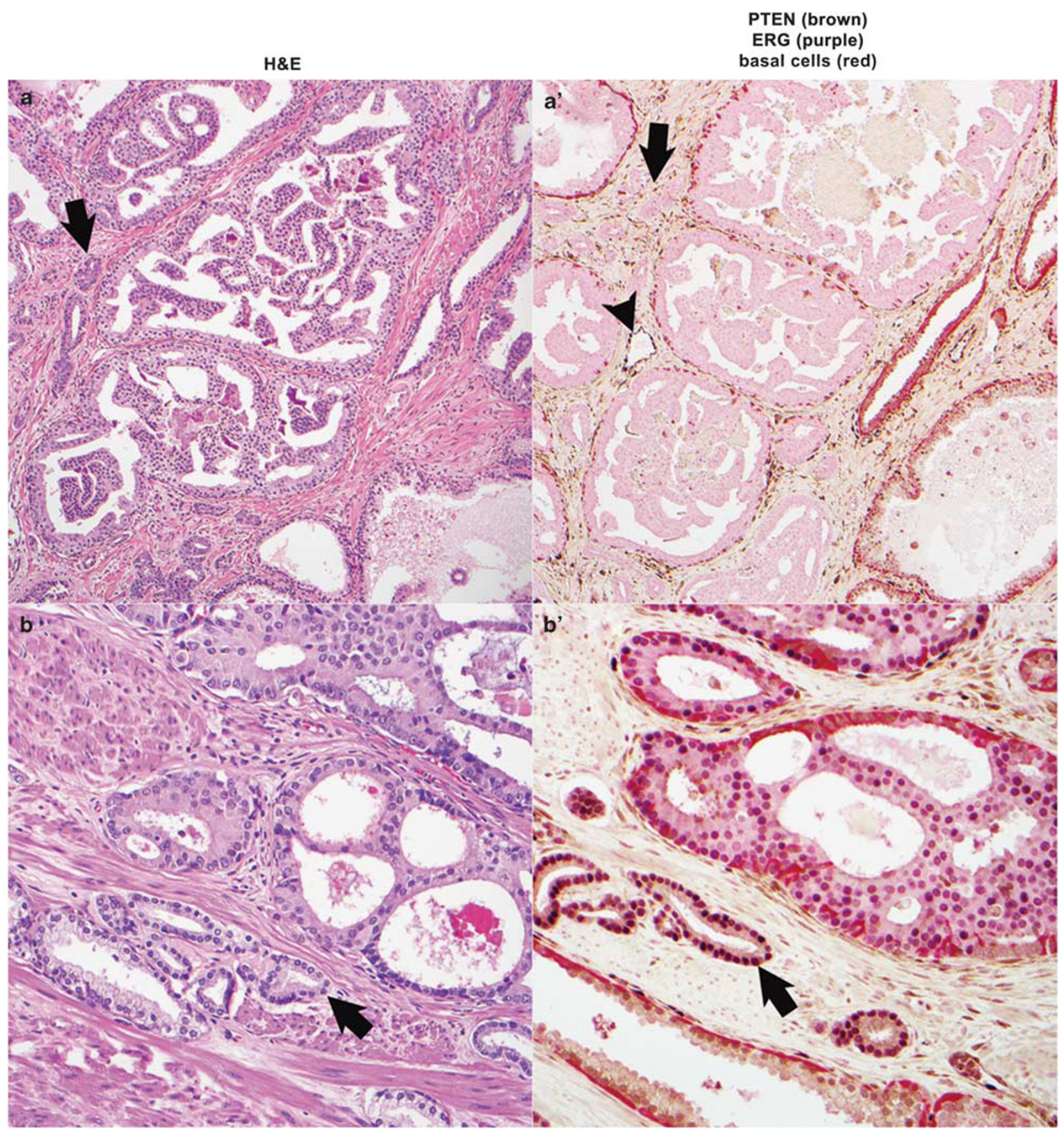

Figure 2 Cytoplasmic PTEN protein loss and nuclear ERG protein expression in intraductal cribriform proliferation. (a, a') Cytoplasmic PTEN protein loss (brown) in micropapillary to loose cribriform intraductal cribriform proliferation. Surrounding invasive tumor demonstrates PTEN loss as well (arrow). ERG protein is not expressed in the tumor cells but is present in the nuclei of surrounding endothelial cells (purple, arrowhead). Basal cells are highlighted by p63-CK903 immunostaining (red). (b, b') Cytoplasmic PTEN protein loss (brown) in intraductal cribriform proliferation, with retention of PTEN protein in surrounding invasive tumor (arrow). Nuclear ERG protein is expressed in the intraductal lesion as well as in the surrounding invasive carcinoma (arrow). (c, c') Cytoplasmic PTEN protein loss in intraductal cribriform proliferation. Surrounding benign luminal cells express high levels of PTEN protein (brown, inset). (d, d') Cytoplasmic PTEN protein loss (brown) with ERG protein expression (purple) in intraductal cribriform proliferation. Surrounding benign glands show lack of ERG expression and retained PTEN, as well as an intact basal cell layer (red, arrow). 


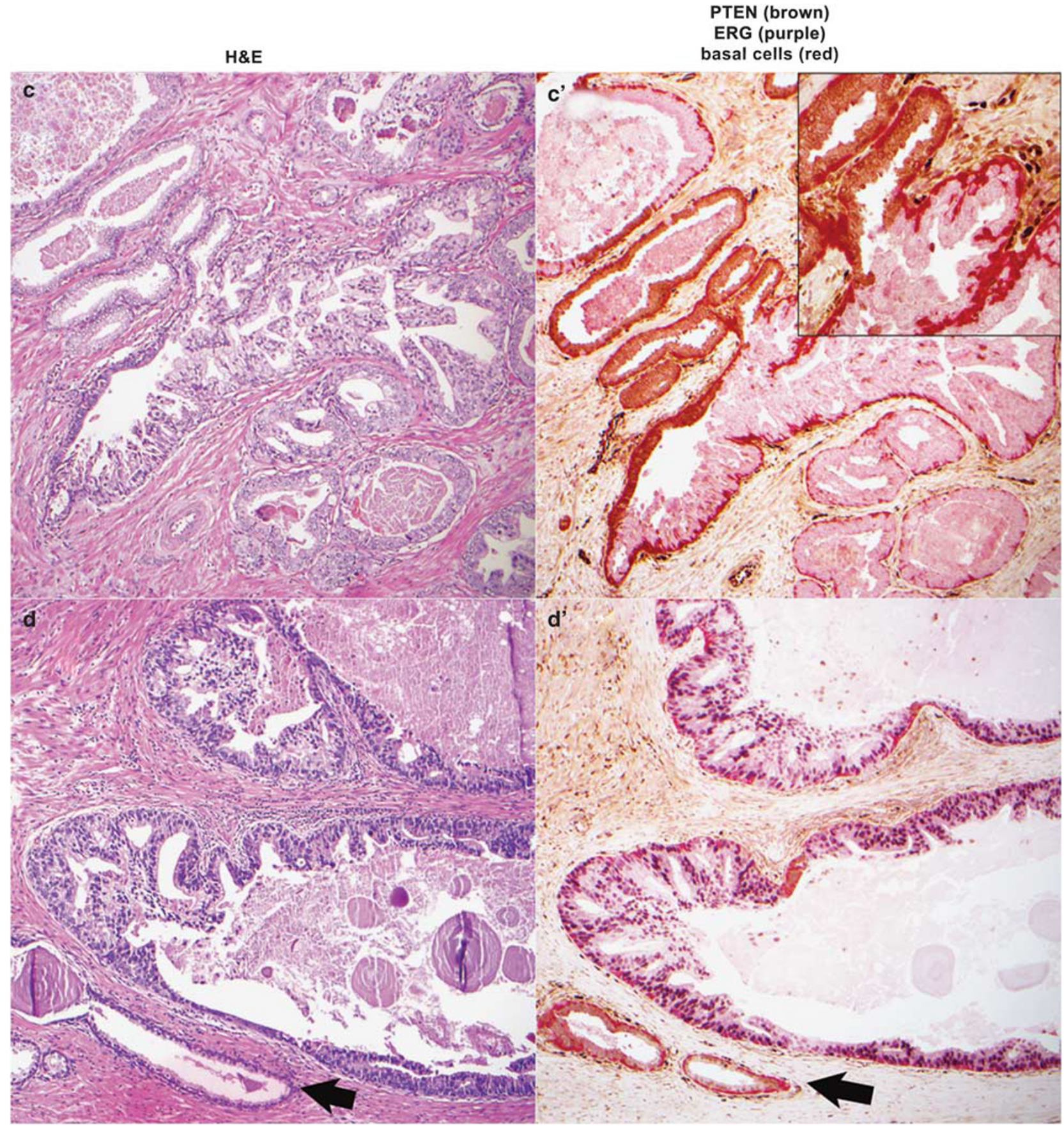

Figure 2 Continued

cytoplasmic expression in $>90 \%$ of lesional cells (Figure 1d). Rates of cytoplasmic PTEN loss were similarly high in intraductal cribriform proliferations, with $87 \%(13 / 15)$ of cases showing total loss and $13 \%(2 / 15)$ of cases showing heterogeneous immunostaining for PTEN with some positive and some negative glands (Table 3a; Figure 2). In contrast, cytoplasmic PTEN protein loss in highgrade PIN was never observed in any case $(0 / 39)$ (Table 3a; Figure 3; $P<0.0001$ compared with rate of homogeneous PTEN loss in intraductal carcinoma cases; Fisher's exact test).

\section{Nuclear PTEN Expression}

Interestingly, preservation of nuclear PTEN immunostaining in a subset of lesional cells was observed frequently in intraductal carcinoma. Of the 29 intraductal carcinoma cases with homogeneous cytoplasmic PTEN protein loss, 62\% (18/29) showed at least focal nuclear positivity for PTEN. Of the 9 intraductal carcinoma cases with mixed PTEN positivity, 44\% (4/9) showed nuclear PTEN positivity ( $P=\mathrm{NS}$; Fisher's exact test). This nuclear expression was easily distinguished from 
Table 3 (a) PTEN protein expression in intraductal lesions. (b) ERG protein expression in intraductal lesions

\begin{tabular}{lrcc}
\hline & $\begin{array}{c}\text { Intraductal } \\
\text { carcinoma }\end{array}$ & $\begin{array}{c}\text { Intraductal } \\
\text { cribriform } \\
\text { proliferation }\end{array}$ & $\begin{array}{c}\text { High- } \\
\text { grade PIN }\end{array}$ \\
\hline \multicolumn{1}{l}{ PTEN positive } & $7(16 \%)$ & $0(0 \%)$ & $39(100 \%)$ \\
PTEN negative & $29(64 \%)$ & $13(87 \%)$ & $0(0 \%)$ \\
Heterogeneous & $9(20 \%)$ & $2(13 \%)$ & $0(0 \%)$ \\
b & & & \\
ERG negative & $19(42 \%)$ & $5(33 \%)$ & $34(87 \%)$ \\
ERG positive & $25(56 \%)$ & $10(67 \%)$ & $1(3 \%)$ \\
Heterogeneous & $1(2 \%)$ & $0(0 \%)$ & $4(10 \%)$
\end{tabular}

cytoplasmic expression, and was most evident in lesional cells situated toward the center of the gland, farthest from the basal cell layer (Figure 4a). In the group of PTEN-negative intraductal carcinoma cases, nuclear PTEN positivity was more frequently observed in ERG-expressing cases (15/19 or $79 \%$ ) and significantly less frequently seen in ERGnegative cases $(3 / 10$ or $33 \% ; P=0.0169$; Fisher's exact test). It was generally easy to distinguish nuclear PTEN (brown; DAB) from nuclear ERG (purple; Vector VIP) in this group because ERG protein expression was frequently decreased in the same subset of cells where nuclear PTEN expression was increased (Figure 4b). Of intraductal cribriform proliferation cases with homogeneous PTEN loss, 100\% (15/15) showed focal nuclear positivity for PTEN.

\section{Association Between Intraductal Lesion Morphology and PTEN Protein Expression}

Of intraductal carcinoma cases with homogeneous PTEN protein loss, $72 \%(21 / 29)$ contained dense cribriform architecture and 65\% showed a component of loose cribriform architecture. Solid architecture was uncommon in PTEN-negative cases $(10 \%$ or $3 / 29)$, but was seen much more commonly in PTEN-positive lesions (57\% or $4 / 7)$. This difference was statistically significant $(P=0.0164$; Fisher's exact test). Micropapillary architecture was uncommon in both groups $(2 \%$ or $1 / 29$ for PTENnegative cases and $14 \%$ or $1 / 7$ for PTEN-positive cases). All PTEN-negative intraductal cribriform proliferation cases were characterized by loose cribriform architecture (100\% or 15/15). Coagulative necrosis was observed at least focally in 48\% (14/29) of the homogeneously PTEN-negative intraductal carcinoma cases vs 14\% (1/7) of the PTEN-positive cases, although this difference did not reach statistical significance ( $P=\mathrm{NS}$; Fisher's exact test). Foamy cytoplasm was seen in $34 \%(10 / 29)$ of the homogeneously PTEN-negative intraductal carcinoma cases and in $20 \%(3 / 15)$ of the homogeneously PTEN-negative intraductal cribriform proliferation cases. In contrast, foamy cytoplasm was not observed in any of the homogeneously PTEN-positive intraductal carcinoma cases $(0 / 7)(P=$ NS; Fisher's exact test).

\section{Nuclear ERG Expression in Intraductal Lesions}

Overall, ERG was expressed in at least a component of the intraductal carcinoma in $58 \%$ of cases $(26 / 45)$, with $56 \%$ uniformly expressing ERG and only 1 case showing mixed ERG positivity in the intraductal carcinoma lesion (Table 3b; Figure 1b). In intraductal cribriform proliferations, ERG was expressed in $67 \%$ of cases (10/15) (Table 3b; Figures 2b and d). Finally, in high-grade PIN, ERG was expressed in 13\% $(5 / 39)$ of cases, with the majority $(4 / 5 ; 80 \%)$ showing ERG expression in only a subset of the glands involved by high-grade PIN lesions (Table 3b; Figure 3). The difference between rates of ERG expression in intraductal carcinoma and high-grade PIN cases was statistically significant $(P<0.0001$; Fisher's exact test $)$.

\section{Concordance of PTEN/ERG Expression Between Invasive and Intraductal Lesions}

Cytoplasmic PTEN and ERG protein status was highly concordant between intraductal tumors and associated invasive tumors in the 43 cases that could be evaluated. Of note, two cases of intraductal carcinoma $(2 / 45,4 \%)$ did not have an associated invasive tumor component in the radical prostatectomy specimen. Of the 37 cases of intraductal carcinoma that showed cytoplasmic PTEN loss in at least a component of the intraductal lesion, $92 \%$ (34/37) showed PTEN loss in at least a component of the concurrent invasive tumor (Table 4a). Of the intraductal carcinoma cases that were PTEN positive, $100 \%$ (6/6) had associated PTEN-positive invasive tumors. Similarly, $100 \%(26 / 26)$ of intraductal carcinoma cases with ERG protein expression also expressed ERG protein in at least a component of the invasive tumor. Of the ERG-negative intraductal carcinoma cases, 100\% (16/16) had ERGnegative invasive tumors as well (Table 5a). For intraductal cribriform proliferations, the concordance for PTEN and ERG between the intraductal lesion and invasive tumor was also very high. Of the intraductal cribriform proliferation cases that had PTEN loss in at least a component of the intraductal lesion, $100 \%$ (15/15) also had PTEN loss in at least a component of the invasive tumor (Table 4b). Similarly, there was $100 \%$ concordance $(15 / 15)$ for ERG status between the intraductal cribriform proliferations and the concurrent invasive tumors (Table 5b). In contrast, there was much less concordance between the high-grade PIN and invasive tumor components in terms of PTEN and ERG status. Of the high-grade PIN cases, 77\% (30/39) had invasive tumor present for evaluation on the same section as the high-grade PIN lesion. Of the PTEN-positive high-grade PIN cases, 83\% (25/30) were associated with PTEN-positive invasive tumors, whereas $17 \%$ 


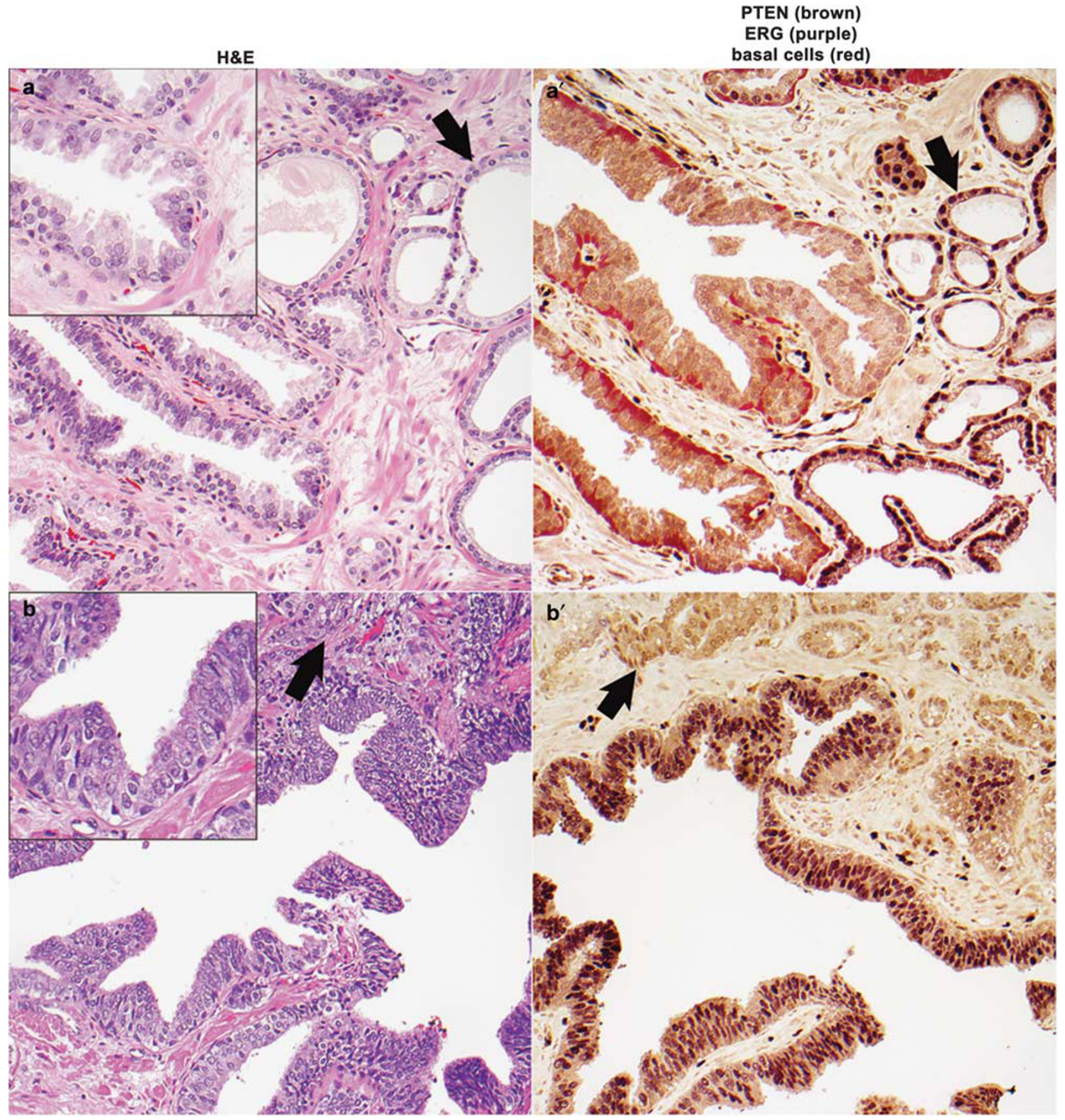

Figure 3 PTEN protein is retained in high-grade PIN lesions. (a, a') PTEN protein (brown) is expressed in high-grade PIN (nucleoli visible in inset), but ERG protein (purple) is expressed only in the surrounding invasive cancer, which lacks a basal cell (red) layer (arrow). (b, b') PTEN (brown) and ERG (purple) protein are both expressed in a high-grade PIN lesion (nucleoli visible in inset), whereas surrounding invasive carcinoma (arrow) expresses PTEN (brown), but not ERG (purple).

(5/30) were associated with PTEN-negative invasive tumors (Table 4c). Similarly, of the ERG-negative high-grade PIN cases, only $62 \% \quad(16 / 26)$ were associated with ERG-negative invasive tumors, whereas $38 \%(10 / 26)$ were associated with ERGpositive invasive tumors. Of the high-grade PIN cases expressing ERG in at least a component, only $25 \%(1 / 4)$ were associated with an ERG-positive invasive tumor, whereas the rest were associated with ERG-negative tumors (Table 5c).

\section{Correlation Between PTEN and ERG Expression} within Intraductal or Invasive Lesions

As previously reported for invasive tumors, ${ }^{45-48}$ there was a trend toward correlation between PTEN and ERG status in homogeneously staining intraductal carcinoma lesions, such that cytoplasmic PTEN protein loss was more common in lesions that expressed ERG protein compared with those without ERG expression, although this did not reach statistical significance $(90 \%$ vs $67 \%$; 


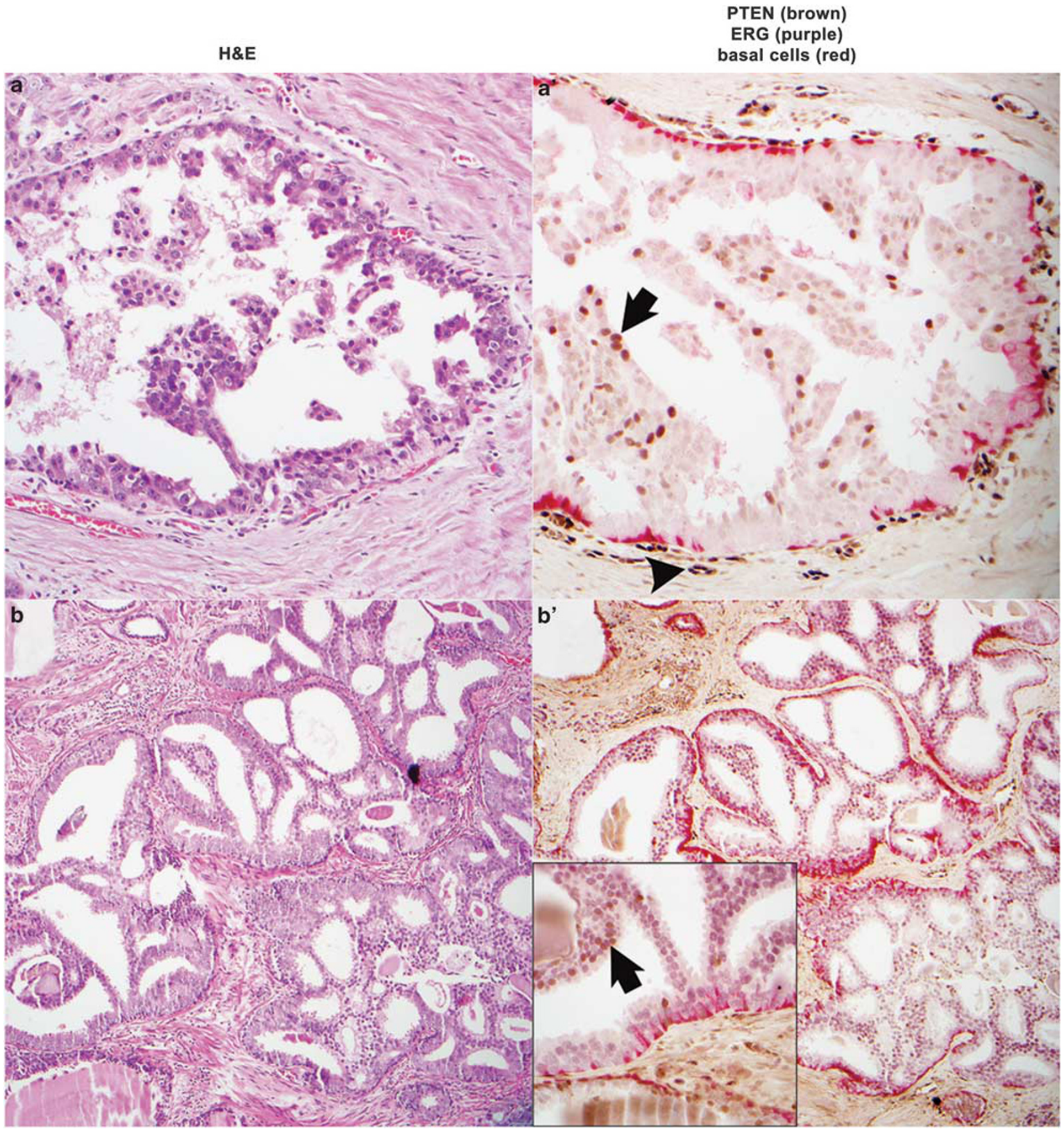

Figure 4 Nuclear PTEN protein is focally expressed in intraductal lesions with cytoplasmic PTEN loss. (a, a') Micropapillary intraductal carcinoma lesion with cytoplasmic PTEN protein loss, but retention of nuclear PTEN, particularly in cells toward the center of the lumen (arrow). ERG (purple) is negative in this case but is visible in surround endothelial cell nuclei (arrowhead). (b, b') Cytoplasmic PTEN protein loss in focally micropapillary intraductal cribriform proliferation with nuclear ERG expression. Focally, nuclear PTEN (brown) is visible in some cells and can be easily distinguished from purple ERG stain (inset, arrow).

Table 6a; $P=0.1028$ by Fisher's exact test). A more striking correlation was evident in the invasive tumors concurrently associated with intraductal carcinoma, intraductal cribriform proliferation or high-grade PIN, with cytoplasmic PTEN protein loss in $67 \%$ of ERG-positive tumors vs $31 \%$ of ERG-negative tumors (Table $6 \mathrm{~d} ; P=0.006$ by Fisher's exact test).

\section{Discussion}

IDC-P Represents the First Identifiable Morphologic Correlate of PTEN Protein Loss

PTEN is one of the most frequently inactivated tumor suppressors in prostate cancer and it remains one of the most powerful single gene prognostic indicators in the disease. Although the majority of 
Table 4 (a) Concordance of PTEN protein status between intraductal carcinoma and invasive tumor. (b) Concordance of PTEN protein status between intraductal cribriform proliferation and invasive tumor. (c) Concordance of PTEN protein status between high-grade PIN and invasive tumor

Cytoplasmic PTEN status of associated invasive tumor

\begin{tabular}{|c|c|c|c|c|}
\hline & $\begin{array}{c}\text { PTEN } \\
\text { positive }\end{array}$ & $\begin{array}{c}\text { PTEN } \\
\text { negative }\end{array}$ & $\begin{array}{l}\text { Hetero- } \\
\text { geneous }\end{array}$ & \\
\hline \multicolumn{5}{|l|}{$a$} \\
\hline \multicolumn{5}{|c|}{ Cytoplasmic PTEN status of intraductal carcinoma } \\
\hline PTEN positive & 6 & 0 & 0 & $P<0.0001$ \\
\hline PTEN negative & 2 & 19 & 7 & \\
\hline Heterogeneous & $9 \begin{array}{c}1 \\
(21 \%)\end{array}$ & $\begin{array}{c}3 \\
22(51 \%)\end{array}$ & $\begin{array}{c}5 \\
12(28 \%)\end{array}$ & \\
\hline \multicolumn{5}{|l|}{$b$} \\
\hline \multicolumn{5}{|c|}{ Cytoplasmic PTEN status of intraductal cribriform proliferation } \\
\hline PTEN positive & 0 & 0 & 0 & \\
\hline PTEN negative & 0 & 5 & 8 & \\
\hline Heterogeneous & $\begin{array}{c}0 \\
0(0 \%)\end{array}$ & $\begin{array}{c}0 \\
5(33 \%)\end{array}$ & $\begin{array}{c}2 \\
10(66 \%)\end{array}$ & \\
\hline \multirow{2}{*}{\multicolumn{5}{|c|}{$\begin{array}{l}\text { C } \\
\text { Cvtoplasmic PTEN status of high-grade PIN }\end{array}$}} \\
\hline & & & & \\
\hline PTEN positive & 25 & 5 & 0 & \\
\hline PTEN negative & 0 & 0 & 0 & \\
\hline Heterogeneous & $\begin{array}{c}0 \\
25(83 \%)\end{array}$ & $\begin{array}{c}0 \\
5(17 \%)\end{array}$ & $\begin{array}{c}0 \\
0(0 \%)\end{array}$ & \\
\hline
\end{tabular}

Table 5 (a) Concordance of ERG protein status between intraductal carcinoma and invasive tumor. (b) Concordance of ERG protein status between intraductal cribriform proliferation and invasive tumor. (c) Concordance of ERG protein status between high-grade PIN and invasive tumor

ERG status of associated invasive tumor

\begin{tabular}{ccc}
\hline ERG & ERG & Hetero- \\
negative & positive & geneous \\
\hline
\end{tabular}

$a$

ERG status of intraductal carcinoma

$\begin{array}{lcccc}\text { ERG negative } & 16 & 0 & 1 & P<0.0001 \\ \text { ERG positive } & 0 & 24 & 1 & \\ \text { Heterogeneous } & 0 & 0 & 1 & \\ & 16(37 \%) & 24(56 \%) & 3(7 \%) & \end{array}$

$b$

ERG status of intraductal cribriform proliferation

ERG negative

ERG positive

Heterogeneous

5
0
0
$5(33 \%)$

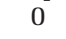

10

$10(66 \%)$

0

$0 \quad P=0.0003$

$5(33 \%)$

$3(7 \%)$

ERG status of high-grade PIN

$\begin{array}{lcccc}\text { ERG negative } & 16 & 10 & 0 & \\ \text { ERG positive } & 0 & 1 & 0 & P=\mathrm{NS} \\ \text { Heterogeneous } & 3 & 0 & 0 & \\ & 19(63 \%) & 11(37 \%) & 0(0 \%) & \end{array}$

previous work has focused on genomic deletions at the PTEN locus, which occur in $17-68 \%$ of surgically resected prostate tumors as detected by FISH, ${ }^{32-34,47,49}$ IHC detection of PTEN protein loss
Table 6 (a) Relationship between PTEN and ERG protein status for homogeneously staining cases of intraductal carcinoma. (b) Relationship between PTEN and ERG protein status for homogeneously staining cases of intraductal cribriform proliferation. (c) Relationship between PTEN and ERG protein status for homogeneously staining cases of HGPIN. (d) Relationship between PTEN and ERG protein status for homogeneously staining cases of invasive carcinoma

\begin{tabular}{lccc}
\hline & $\begin{array}{c}\text { ERG } \\
\text { negative }\end{array}$ & $\begin{array}{c}\text { ERG } \\
\text { positive }\end{array}$ & \\
\hline $\begin{array}{l}\text { Intraductal carcinoma } \\
\quad \text { PTEN positive }\end{array}$ & 5 & & \\
$\quad$ PTEN negative & 10 & 19 & \\
& & & \\
Intraductal cribriform & proliferation & & \\
$\quad$ PTEN positive & 0 & 0 & \\
$\quad$ PTEN negative & 5 & 8 & \\
$\quad$ & & & \\
C High-grade PIN & & & \\
$\quad$ PTEN positive & 34 & 1 & \\
$\quad$ PTEN negative & 0 & 0 & \\
$\quad$ Invasive carcinoma & & & \\
$\quad$ PTEN positive & 22 & 11 & \\
$\quad$ PTEN negative & 10 & 22 & \\
\hline
\end{tabular}

may be a more sensitive assay. Although a number of previous studies have used IHC to detect PTEN genomic loss, the sensitivity of these assays has generally been low, in large part because the antibodies and assays used have been insufficiently validated (reviewed in Lotan et $a l^{35}$ ). Recently, along with others, we have taken advantage of newly available reliable PTEN monoclonal antibodies as well as cell line and tissue genetic controls and demonstrated that that PTEN protein detection by IHC sensitively detects genomic PTEN loss. ${ }^{35,41}$ In a panel of 58 cell lines, we found that PTEN IHC was $100 \%$ sensitive and $97.8 \%$ specific for detection of genomic PTEN loss. ${ }^{35}$ In an additional 119 prostate tumor cases, PTEN protein loss detected $75 \%$ and $86 \%$ of cases with PTEN genomic loss, as detected by FISH or high-resolution SNP microarray, respectively. ${ }^{35}$ Further, in a tissue microarray (TMA) study of 263 prostate cancer patients treated by radical prostatectomy, we demonstrated that the frequency of cytoplasmic PTEN protein loss was highly correlated with Gleason grade and pathologic stage, and ranged up to $45 \%$ in the highest-risk cases (Gleason $8-10$ or pT3B or distant metastases). ${ }^{35}$

Surprisingly, although analyzing the data from this study, we observed that the rate of PTEN loss in TMA spots containing intraductal carcinoma approached 100\% (13/13 cases, AMD, TLL, B Gurel, unpublished data). Intrigued that the rate of PTEN protein loss in intraductal carcinoma appeared to be nearly double that of our most advanced distant metastatic samples, we set out to systematically 
study the rate of PTEN protein loss in a larger group of intraductal carcinoma cases. Here, using established morphologic criteria to identify intraductal carcinoma and standard tissue sections to allow for assessment of staining heterogeneity, we demonstrate that $84 \%(38 / 45)$ of intraductal carcinoma cases have at least focal cytoplasmic PTEN protein loss and virtually all of these cases have associated invasive tumor with PTEN protein loss as well. Importantly, the presence of intraductal carcinoma is an extremely strong predictor of PTEN protein loss in the invasive tumor, with $79 \%$ of cases with intraductal carcinoma containing at least heterogeneous PTEN loss in the invasive tumor compared with $17 \%$ of roughly stage and grade-matched cases containing high-grade PIN, but not intraductal carcinoma. These data strongly suggest that the presence of intraductal carcinoma is a sensitive indicator of an invasive tumor with underlying PTEN loss, making intraductal carcinoma the first described morphologic correlate of this molecular aberration in prostate cancer.

\section{PTEN Loss in Intraductal Carcinoma may Provide a Potential Explanation for Strong Association of Intraductal Carcinoma with Poor Prognosis in Prostate Cancer}

As its early description, pathologists have recognized that morphologically identifiable intraductal carcinoma is strongly associated with poor prognosis in prostate cancer. In radical prostatectomy specimens, intraductal carcinoma is commonly seen in cases with invasive tumor of high pathologic stage and Gleason score, and has been shown to be independently associated with reduced time to biochemical progression. ${ }^{3-5}$ Indeed, in our own retrospectively identified cohort of intraductal carcinoma, $>80 \%$ of cases contained invasive tumor at pT3A or higher and Gleason 7 or higher. When identified on biopsy with associated invasive tumor, intraductal carcinoma predicted for early biochemical recurrence following radiotherapy, even after correcting for Gleason score. ${ }^{10}$ Similarly, in a surgical cohort, presence of intraductal carcinoma on biopsy was correlated with a worse pathologic outcome at radical prostatectomy than would have been predicted based on the Partin tables alone. ${ }^{11}$ Finally, the presence of isolated intraductal carcinoma on biopsy without sampled invasive tumor is almost invariably associated with invasive tumor of high-grade and stage at radical prostatectomy. $^{9}$ Similarly, PTEN genomic and PTEN protein loss in prostate cancer have been associated with decreased time to metastasis and independently associated with decreased time to biochemical recurrence in surgical cohorts. ${ }^{34,35,50,51}$ Given our finding that intraductal carcinoma is essentially a morphologic marker of PTEN loss in prostate cancer, it is possible that PTEN loss is a key underlying molecular aberration driving poor prognosis in intraductal carcinoma.

\section{Intraductal Carcinoma Exists on a Morphologic Spectrum and Morphologic Criteria Lack Sensitivity Because they are Highly Specific}

Despite its clear association with clinically aggressive disease, reliable identification of intraductal carcinoma based on morphologic criteria alone has proven difficult. This is in large part because intraductal carcinoma may overlap morphologically with the more common non-cribriform high-grade PIN $^{8}$ and the less common cribriform high-grade PIN. Given the disparate clinical management of intraductal carcinoma and high-grade PIN (the presence of isolated intraductal carcinoma on biopsy merits definitive surgical or radio-therapy, ${ }^{8}$ whereas the presence of isolated high-grade PIN may not even require a follow-up biopsy ${ }^{52}$ ), accurately distinguishing between the two in biopsy specimens is paramount. To date, a number of morphologic classification schemes have been proposed to help distinguish intraductal carcinoma from high-grade PIN, most of which are fairly stringent to avoid over-diagnosis of intraductal carcinoma with subsequent over-treatment. ${ }^{6-8}$ However, there is little question that even after using such criteria, there remain a substantial group of intraductal proliferative lesions that are more concerning than ordinary high-grade PIN, but that do not meet criteria for intraductal carcinoma (herein referred to as intraductal cribriform proliferations). In fact, in 2011 on a busy urologic pathology consultation service, we made the diagnosis of intraductal cribriform proliferation on biopsy nearly 1.5 times as frequently as we diagnosed isolated intraductal carcinoma (JIE, TLL, unpublished data), reflecting a tendency toward the conservative management of lesions where the correct classification is unclear.

\section{Combined Immunostaining for PTEN and Basal Cell Markers Reliably Distinguishes Morphologically Identified Intraductal Carcinoma from High-grade PIN}

Perhaps the most clinically significant finding in the current study is that the presence of cytoplasmic PTEN protein loss sensitively identifies intraductal carcinoma cases meeting morphologic criteria and accurately distinguishes them from isolated highgrade PIN, where PTEN protein loss is essentially never observed. In contrast, immunolabeling for ERG was less sensitive and specific than PTEN loss for identifying intraductal carcinoma cases, and was occasionally seen in high-grade PIN. Thus, in the case of reliable identification of cytoplasmic PTEN protein loss, ERG immunostaining may not add much to resolve this important differential diagnosis. Furthermore (and consistent with prior studies) 
we observed a trend toward a positive correlation between PTEN loss and ERG expression, ${ }^{45-48}$ which means that cases with PTEN protein retention and ERG expression were relatively rare. One limitation to the current study is that it was carried out using standard tissue sections from radical prostatectomy specimens. Given that nearly one-third of the intraductal carcinoma lesions we analyzed showed heterogeneous PTEN immunolabeling, and that biopsy tissue samples are considerably smaller, we would expect the sensitivity of PTEN loss as a marker for intraductal carcinoma would be somewhat lower in prostate needle biopsy specimens. Indeed, studies to assess this possible shortcoming are currently underway. However, as PTEN protein loss has such a high sensitivity for intraductal carcinoma detection in the current study, we anticipate that PTEN labeling will continue to be useful in the biopsy setting as well, even if the sensitivity is somewhat decreased.

\section{Previous Studies have Found a Low Rate of PTEN Gene Deletion in High-grade PIN, however, these Deletions Appear Specific for High-grade PIN Immediately Adjacent to Invasive Tumor Foci}

Although only a very small number of studies have addressed this question previously, at least two studies did find evidence of rare PTEN deletion by FISH in high-grade PIN lesions. ${ }^{33,47}$ Superficially, these studies appear to contrast with our finding in the current study that PTEN protein loss is never observed in isolated high-grade PIN. Interestingly, in the paper by Han et al, ${ }^{47}$ the authors found the rate of PTEN deletion by FISH to be $9 \%$ (3/33). However, a significant caveat of this study is that all of the PIN foci harboring PTEN deletion were found within $3 \mathrm{~mm}$ of an adjacent invasive tumor. In contrast, in isolated high-grade PIN cases $(>3 \mathrm{~mm}$ from surrounding tumor), no PTEN deletions were identified. ${ }^{47}$ As the majority (59\%) of our highgrade PIN cases were $>3 \mathrm{~mm}$ from surrounding adjacent tumor in the current study, our data are potentially consistent with Han et al, and strongly suggest that some of the apparent high-grade PIN cases immediately adjacent to invasive tumor may actually represent intraductal carcinoma that does not meet current morphologic criteria. Although Yoshimoto et $a l^{33}$ does not specify whether the highgrade PIN lesions they analyzed were exclusively adjacent to invasive tumor, the figures suggest that at least a subset were immediately adjacent to invasive tumor. Again, PTEN loss in a minority of these highgrade PIN cases may be consistent with intraductal spread of high-grade prostate cancer that is not morphologically recognized by current criteria. In order to exclude inclusion of under-recognized intraductal carcinoma from our high-grade PIN group, we focused on high-grade PIN lesions $>3 \mathrm{~mm}$ from adjacent invasive tumors. Ultimately, this may be why the rate of PTEN loss (and the rate of ERG expression) we have reported in highgrade PIN is somewhat lower than previously reported. ${ }^{33,47}$

The molecular profile of intraductal carcinoma and intraductal cribriform proliferation is strongly concordant with concurrent invasive carcinoma: as its initial description, pathologists have debated whether intraductal carcinoma represents advanced invasive carcinoma that has begun to spread along existing prostatic ducts, or whether it represents a precursor lesion, akin to a very advanced form of high-grade PIN. To address this question, two prior studies have investigated rates of $\mathrm{LOH}$ in high-grade PIN vs intraductal carcinoma and invasive tumor, either by array comparative genomic hybridization or using a panel of 12 microsatellite loci, finding that intraductal carcinoma more closely resembled invasive tumor than high-grade PIN, with high rates of LOH at multiple loci. ${ }^{13,14}$ Similarly, recent work looking at TMPRSS2-ERG rearrangement has also found that rates of rearrangement in intraductal carcinoma more closely approximated that of invasive tumor than high-grade PIN, and has shown that the concordance between intraductal carcinoma and concurrent invasive tumors is high. ${ }^{15}$ Our data investigating PTEN and ERG status in intraductal carcinoma and concurrent invasive tumors adds to this body of work and further confirms that intraductal carcinoma is likely genetically concordant at these loci with the surrounding invasive tumors in the majority of cases. In contrast, high-grade PIN showed a considerably lower concordance rate with invasive carcinoma in terms of PTEN status, suggesting that PTEN deletion is a relatively late event during the prostate cancer progression sequence. Combined with recent data suggesting that isolated intraductal carcinoma without invasive tumor in radical prostatectomy specimens is quite rare, the weight of the evidence supports the hypothesis that in the vast majority of cases, intraductal carcinoma represents retrograde spread of invasive tumor into pre-existing prostatic ducts rather than a de novo lesion.

\section{The High Rate of Loss of Cytoplasmic PTEN in Intraductal Cribriform Proliferations Suggests that Many of these Lesions are Actually a Form of Intraductal Carcinoma not Recognized by Current Morphologic Criteria}

Interestingly, we also found an extremely high rate of concordance between PTEN and ERG status in intraductal cribriform proliferations and the concurrent invasive tumor. This finding, combined with the uniform PTEN protein loss in these lesions, strongly suggests that the intraductal cribriform proliferations included in this study likely represent a form of intraductal carcinoma that is not recognized by the current morphologic criteria. Of note, 
all intraductal cribriform proliferations included in this study had very similar morphologic features, including loose cribriform architecture but lacking marked cytologic atypia and comedonecrosis, which excluded them from the intraductal carcinoma group based on the morphologic criteria that we used. ${ }^{8}$ Importantly, because all of these lesions were intimately associated with concurrent invasive tumor, other groups, such as Zhou et al, would likely have classified these cases as a form of intraductal carcinoma based on morphology alone. ${ }^{7}$ Although this classification scheme has been proposed for use in radical prostatectomy specimens, it remains unclear how these lesions should be classified in biopsy specimens, where the concurrent invasive tumor may not be sampled. In these cases, evaluation of PTEN protein expression, along with basal cell markers, may be particularly useful in determining whether to recommend definitive treatment for these lesions. Using a cohort of isolated intraductal cribriform proliferations on biopsy with clinical follow-up, we are currently evaluating the utility of PTEN IHC in this setting.

\section{The Mechanism of Retained Nuclear PTEN Protein Expression in Intraductal Carcinoma Lesions with Cytoplasmic PTEN Loss Remains Unclear}

Despite the dramatic loss of cytoplasmic PTEN protein in intraductal carcinoma and intraductal cribriform proliferations, one notable finding in this study was the heterogeneous retention of nuclear PTEN protein in at least some tumor cell nuclei in the majority of cases with cytoplasmic protein loss. Although the role of cytoplasmic PTEN has been clear for some time, the role of nuclear PTEN in tumor suppression has only recently been elucidated, and may be cell-type specific. ${ }^{53}$ In benign prostate cells, PTEN is clearly present in both the cytoplasm and in the nucleus. However, in our extensive study of prostate adenocarcinomas, we have never observed a case where nuclear PTEN was lost and cytoplasmic PTEN was preserved, suggesting that the main tumor suppressive effects of PTEN in prostate cancer are likely carried out in the cytoplasm. ${ }^{35}$ One interesting possibility is that nuclear sequestration of PTEN protein in intraductal carcinoma cases may actually be an alternative mechanism of PTEN tumor-suppressor inactivation. In support of this, there is evidence that some naturally occurring syndromic and cancer-associated mutations in a highly conserved N-terminal region of PTEN lead to nuclear sequestration of the protein and impair its growth suppressive effects while preserving in vitro lipid phosphatase activity. ${ }^{54}$ Alternatively, it is possible that nuclear PTEN accumulation reflects specific instability of PTEN protein in the cytoplasm, perhaps because of mutations leading to structural changes and subsequent degradation in the cytoplasmic proteasome, whereas mutant nuclear PTEN remains relatively protected. Finally, nuclear PTEN localization can also be seen in a number of cell types following ATP depletion. ${ }^{55}$ Strikingly, nuclear PTEN protein was expressed specifically in the cells toward the center of the involved gland (ie, off of the extracellular matrix) in intraductal carcinoma lesions, a location that entails substantial displacement from the stromal-based blood supply and likely results in hypoxia and ATP-depletion.

\section{The Retention of Nuclear PTEN in Intraductal Carcinoma Lesions with Cytoplasmic PTEN Loss Provides Potential Insight into the Mechanism of PTEN Inactivation in these Lesions}

Whatever the mechanism of nuclear PTEN retention in the face of cytoplasmic depletion, the presence of nuclear PTEN in intraductal carcinoma cases means it is highly likely that at least one allele of PTEN remains intact in the majority of these lesions. Although genomic deletions are the most common recognized cause of PTEN loss in prostate cancer, our recent study comparing PTEN protein expression and PTEN genomic status suggests that alternative mechanisms for PTEN protein loss are much more common than previously thought. In that study, we found that close to $40 \%$ of invasive tumors with PTEN protein loss did not show PTEN genomic loss as detectable by FISH or high-resolution SNP (single-nucleotide polymorphism) microarray. ${ }^{35}$ In addition, we found that PTEN IHC was as sensitive for the detection of hemizygous PTEN loss by SNP array as it was for the detection of homozygous loss. In the current study, the finding that nuclear PTEN persists in the absence of cytoplasmic PTEN in $70 \%$ of cases strongly suggests that PTEN genomic loss in these cases is at most hemizygous, with alternative mechanisms accounting for cytoplasmic PTEN protein loss in the majority of intraductal carcinoma. In this case, it is also possible, and perhaps likely given that only nuclear staining occurs, that the retained PTEN allele is mutated (eg, small insertions or deletions) in a manner that would not be detectable by FISH or SNP microarrays.

In conclusion, we have found that cytoplasmic PTEN protein loss occurs in the majority of morphologically identified intraductal carcinoma cases and is never observed in isolated high-grade PIN lesions. This makes the presence of intraductal carcinoma the first identifiable morphologic correlate of PTEN loss in prostate cancer, and provides the first plausible specific molecular explanation for why intraductal carcinoma is strongly associated with poor prognosis in prostate cancer. Although ERG protein expression is present in a subset of intraductal carcinoma and intraductal cribriform proliferation cases, it is occasionally seen in high- 
grade PIN as well, and is less reliable as a marker to distinguish these lesions. Consistent with previous data, the high concordance between PTEN and ERG status in the intraductal lesions and the associated concurrent invasive carcinoma suggests that, at least in the majority of cases, intraductal carcinoma most likely represents intraductal spread of advanced invasive prostate cancer. Importantly, we have also shown that the detection of cytoplasmic PTEN loss helps to definitively classify as intraductal carcinoma a group of lesions where the differential diagnosis based on morphology alone is between intraductal carcinoma and high-grade PIN. Future studies will focus on confirming the utility of PTEN IHC for distinguishing intraductal carcinoma from high-grade PIN in the prostate biopsy setting, both in the presence and in the absence of sampled invasive tumor.

\section{Acknowledgements}

Funding for this research was provided in part by the Prostate Cancer Foundation Young Investigator Award (TLL), the NIH/NCI Prostate SPORE P50CA58236 and a generous gift from Mr David $\mathrm{H}$ Koch (AMD).

\section{Disclosure/conflict of interest}

AMD is currently an employee of Predictive Biosciences. However, no funding or other support was provided by the company for any of the work in the manuscript. The terms of the relationship between AMD and Predictive Biosciences are managed by the Johns Hopkins University in accordance with its conflict of interest policies.

\section{References}

1 Kovi J, Jackson MA, Heshmat MY. Ductal spread in prostatic carcinoma. Cancer 1985;56:1566-1573.

2 McNeal JE, Yemoto CE. Spread of adenocarcinoma within prostatic ducts and acini: morphologic and clinical correlations. Am J Surg Pathol 1996;20: 802-814.

3 Wilcox G, Soh S, Chakraborty S, et al. Patterns of highgrade prostatic intraepithelial neoplasia associated with clinically aggressive prostate cancer. Hum Pathol 1998;29:1119-1123.

4 Rubin MA, de La Taille A, Bagiella E, et al. Cribriform carcinoma of the prostate and cribriform prostatic intraepithelial neoplasia: incidence and clinical implications. Am J Surg Pathol 1998;22:840-848.

5 Cohen RJ, McNeal JE, Baillie T. Patterns of differentiation and proliferation in intraductal carcinoma of the prostate: significance for cancer progression. Prostate 2000;43:11-19.

6 Cohen RJ, Wheeler TM, Bonkhoff H, et al. A proposal on the identification, histologic reporting, and implications of intraductal prostatic carcinoma. Arch Pathol Lab Med 2007;131:1103-1109.
7 Shah RB, Magi-Galluzzi C, Han B, et al. Atypical cribriform lesions of the prostate: relationship to prostatic carcinoma and implication for diagnosis in prostate biopsies. Am J Surg Pathol 2010;34:470-477.

8 Guo CC, Epstein JI. Intraductal carcinoma of the prostate on needle biopsy: histologic features and clinical significance. Mod Pathol 2006;19:1528-1535.

9 Robinson BD, Epstein JI. Intraductal carcinoma of the prostate without invasive carcinoma on needle biopsy: emphasis on radical prostatectomy findings. J Urol 2010;184:1328-1333.

10 Van der Kwast T, Al Daoud N, Collette L, et al. Biopsy diagnosis of intraductal carcinoma is prognostic in intermediate and high risk prostate cancer patients treated by radiotherapy. Eur J Cancer 2012;48: 1318-1325.

11 Watts K, Li J, Magi-Galluzzi C, et al. Incidence and clinicopathological characteristics of intraductal carcinoma of the prostate detected in prostate biopsies: a prospective cohort study. Mod Pathol 2012;25:250A.

12 Herawi M, Epstein JI. Immunohistochemical antibody cocktail staining (p63/HMWCK/AMACR) of ductal adenocarcinoma and gleason pattern 4 cribriform and noncribriform acinar adenocarcinomas of the prostate. Am J Surg Pathol 2007;31:889-894.

13 Dawkins HJ, Sellner LN, Turbett GR, et al. Distinction between intraductal carcinoma of the prostate (IDC-P), high-grade dysplasia (PIN), and invasive prostatic adenocarcinoma, using molecular markers of cancer progression. Prostate 2000;44:265-270.

14 Bettendorf O, Schmidt $\mathrm{H}$, Staebler A, et al. Chromosomal imbalances, loss of heterozygosity, and immunohistochemical expression of TP53, RB1, and PTEN in intraductal cancer, intraepithelial neoplasia, and invasive adenocarcinoma of the prostate. Genes Chromosomes Cancer 2008;47:565-572.

15 Han B, Suleman K, Wang L, et al. ETS gene aberrations in atypical cribriform lesions of the prostate: implications for the distinction between intraductal carcinoma of the prostate and cribriform high-grade prostatic intraepithelial neoplasia. Am J Surg Pathol 2010;34: 478-485.

16 Tomlins SA, Rhodes DR, Perner S, et al. Recurrent fusion of TMPRSS2 and ETS transcription factor genes in prostate cancer. Science 2005;310:644-648.

$17 \mathrm{Tu}$ JJ, Rohan S, Kao J, et al. Gene fusions between TMPRSS2 and ETS family genes in prostate cancer: frequency and transcript variant analysis by RT-PCR and FISH on paraffin-embedded tissues. Mod Pathol 2007;20:921-928.

18 Perner S, Demichelis F, Beroukhim R, et al. TMPRSS2:ERG fusion-associated deletions provide insight into the heterogeneity of prostate cancer. Cancer Res 2006;66:8337-8341.

19 Attard G, Clark J, Ambroisine L, et al. Transatlantic Prostate Group. Duplication of the fusion of TMPRSS2 to ERG sequences identifies fatal human prostate cancer. Oncogene 2008;27:253-263.

20 Demichelis F, Fall K, Perner S, et al. TMPRSS2:ERG gene fusion associated with lethal prostate cancer in a watchful waiting cohort. Oncogene 2007;26:4596-4599.

21 Fine SW, Gopalan A, Al-Ahmadie HA, et al. Does TMPRSS2-ERG gene fusion status in prostate cancer correlate with gleason score? Mod Pathol 2008;21:156A.

22 Fine SW, Gopalan A, Leversha MA, et al. TMPRSS2ERG gene fusion is associated with low gleason scores 
and not with high-grade morphological features. Mod Pathol 2010;23:1325-1333.

23 Gopalan A, Leversha MA, Satagopan JM, et al. TMPRSS2-ERG gene fusion is not associated with outcome in patients treated by prostatectomy. Cancer Res 2009;69:1400-1406.

24 Toubaji A, Albadine R, Meeker AK, et al. Increased gene copy number of ERG on chromosome 21 but not TMPRSS2-ERG fusion predicts outcome in prostatic adenocarcinomas. Mod Pathol 2011;24:1511-1520.

25 Cairns P, Okami K, Halachmi S, et al. Frequent inactivation of PTEN/MMAC1 in primary prostate cancer. Cancer Res 1997;57:4997-5000.

$26 \mathrm{Li} \mathrm{J}$, Yen C, Liaw D, et al. PTEN, a putative protein tyrosine phosphatase gene mutated in human brain, breast, and prostate cancer. Science 1997;275: 1943-1947.

27 Steck PA, MA Pershouse, Jasser SA, et al. Identification of a candidate tumour suppressor gene, MMAC1, at chromosome 10q23.3 that is mutated in multiple advanced cancers. Nat Genet 1997;15:356-362.

28 Teng DH, Hu R, Lin H, et al. MMAC1/PTEN mutations in primary tumor specimens and tumor cell lines. Cancer Res 1997;57:5221-5225.

29 Feilotter HE, Nagai MA, Boag AH, et al. Analysis of PTEN and the $10 q 23$ region in primary prostate carcinomas. Oncogene 1998;16:1743-1748.

30 Pesche S, Latil A, Muzeau F, et al. PTEN/MMAC1/ TEP1 involvement in primary prostate cancers Oncogene 1998;16:2879-2883.

31 Wang SI, Parsons R, Ittmann M. Homozygous deletion of the PTEN tumor suppressor gene in a subset of prostate adenocarcinomas. Clin Cancer Res 1998;4:811-815.

32 Verhagen PC, van Duijn PW, Hermans KG, et al. The PTEN gene in locally progressive prostate cancer is preferentially inactivated by bi-allelic gene deletion. J Pathol 2006;208:699-707.

33 Yoshimoto M, Cutz JC, Nuin PA, et al. Interphase FISH analysis of PTEN in histologic sections shows genomic deletions in $68 \%$ of primary prostate cancer and $23 \%$ of high-grade prostatic intra-epithelial neoplasias. Cancer Genet Cytogenet 2006;169:128-137.

34 Yoshimoto M, Cunha IW, Coudry RA, et al. FISH analysis of 107 prostate cancers shows that PTEN genomic deletion is associated with poor clinical outcome. Br J Cancer 2007;97:678-685.

35 Lotan TL, Gurel B, Sutcliffe S, et al. PTEN protein loss by immunostaining: analytic validation and prognostic indicator for a high risk surgical cohort of prostate cancer patients. Clin Cancer Res 2011;17:6563-6573.

36 Mosquera JM, Perner S, Genega EM, et al. Characterization of TMPRSS2-ERG fusion high-grade prostatic intraepithelial neoplasia and potential clinical implications. Clin Cancer Res 2008;14:3380-3385.

37 Zhang S, Pavlovitz B, Tull J, et al. Detection of TMPRSS2 gene deletions and translocations in carcinoma, intraepithelial neoplasia, and normal epithelium of the prostate by direct fluorescence in situ hybridization. Diagn Mol Pathol 2010;19: 151-156.

38 Yaskiv O, Zhang X, Simmerman K, et al. The utility of ERG/P63 double immunohistochemical staining in the diagnosis of limited cancer in prostate needle biopsies. Am J Surg Pathol 2011;35:1062-1068.
39 Furusato B, Tan SH, Young D, et al. ERG oncoprotein expression in prostate cancer: clonal progression of ERG-positive tumor cells and potential for ERG-based stratification. Prostate Cancer Prostatic Dis 2010;13:228-237.

40 Park K, Tomlins SA, Mudaliar KM, et al. Antibodybased detection of ERG rearrangement-positive prostate cancer. Neoplasia 2010;12:590-598.

41 Sangale Z, Prass C, Carlson A, et al. A robust immunohistochemical assay for detecting PTEN expression in human tumors. Appl Immunohistochem Mol Morphol 2011;19:173-183.

42 Chaux A, Albadine R, Toubaji A, et al. Immunohistochemistry for ERG expression as a surrogate for TMPRSS2-ERG fusion detection in prostatic adenocarcinomas. Am J Surg Pathol 2011;35:1014-1020.

43 Kibel AS, Rosenbaum E, Kattan MW, et al. Adjuvant weekly docetaxel for patients with high risk prostate cancer after radical prostatectomy: a multi-institutional pilot study. J Urol 2007;177:1777-1781.

44 McNeal JE, Yemoto CE. Spread of adenocarcinoma within prostatic ducts and acini. morphologic and clinical correlations. Am J Surg Pathol 1996;20:802-814.

45 King JC, Xu J, Wongvipat J, et al. Cooperativity of TMPRSS2-ERG with PI3-kinase pathway activation in prostate oncogenesis. Nat Genet 2009;41:524-526.

46 Carver BS, Tran J, Gopalan A, et al. Aberrant ERG expression cooperates with loss of PTEN to promote cancer progression in the prostate. Nat Genet 2009;41:619-624.

47 Han B, Mehra R, Lonigro RJ, et al. Fluorescence in situ hybridization study shows association of PTEN deletion with ERG rearrangement during prostate cancer progression. Mod Pathol 2009;22:1083-1093.

48 Bismar TA, Yoshimoto M, Vollmer RT, et al. PTEN genomic deletion is an early event associated with ERG gene rearrangements in prostate cancer. BJU Int 2011;107:477-485.

49 Sircar K, Yoshimoto M, Monzon FA, et al. PTEN genomic deletion is associated with p-akt and AR signalling in poorer outcome, hormone refractory prostate cancer. J Pathol 2009;218:505-513.

50 Yoshimoto M, Joshua AM, Cunha IW, et al. Absence of TMPRSS2:ERG fusions and PTEN losses in prostate cancer is associated with a favorable outcome. Mod Pathol 2008;21:1451-1460.

51 Chaux A, Peskoe SB, Gonzalez-Roibon N, et al. Loss of PTEN expression is associated with increased risk of recurrence after prostatectomy for clinically localized prostate cancer. Mod Pathol 2012;25:1543-1549; advance online publication.

52 Epstein JI, Herawi M. Prostate needle biopsies containing prostatic intraepithelial neoplasia or atypical foci suspicious for carcinoma: implications for patient care. J Urol 2006;175:820-834.

53 Shen WH, Balajee AS, Wang J, et al. Essential role for nuclear PTEN in maintaining chromosomal integrity. Cell 2007;128:157-170.

54 Denning G, Jean-Joseph B, Prince C, et al. A short N-terminal sequence of PTEN controls cytoplasmic localization and is required for suppression of cell growth. Oncogene 2007;26:3930-3940.

55 Lobo GP, Waite KA, Planchon SM, et al. ATP modulates PTEN subcellular localization in multiple cancer cell lines. Hum Mol Genet 2008;17:2877-2885. 